\title{
PHY and MAC, cross-layer optimization and design
}

\begin{abstract}
This document gives some definitions about PHY and MAC layer displaying its internal architecture and major functions to both layers. And as the same time states some information about cross-layer design, discussing the architecture of the cross-layer, and focusing on major methods of interfacing with giving one example of data transmission and interfacing which is meant to be very useful in this stage. Each researcher may walk through some problems during time of enhancement and optimization for the cross-layer, so here we stated some challenges to solve some problems with concentrating on number of points of classifications for cross-layer solutions.
\end{abstract}

Keyword: PHY layer; MAC layer; Cross-layer optimization; Architecture and designe 